\title{
Analysis of edema fluids and histologic features of the lung in reexpansion pulmonary edema during video-assisted thoracoscopic surgery
}

\author{
Satoshi Suzuki, MD, ${ }^{a}$ Hiromichi Niikawa, MD, ${ }^{a}$ Jotaro Shibuya, MD, ${ }^{a}$ Tomoko Hosaka, MD, ${ }^{\text {a }}$ Sumiko Maeda, MD, \\ Takashi Suzuki, MD, ${ }^{\text {b }}$ and Masashi Handa, MD, a Sendai, Japan
}

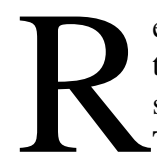
eexpansion pulmonary edema $(\mathrm{PE})$ is a rare complication of general thoracic surgery; however, it may lead to severe hypoxemia with a mortality as high as $20 \%$. $^{1}$ The exact mechanisms of development and resolution of reexpansion PE remain undefined. We present here 2 cases of reexpansion $\mathrm{PE}$ in which edema fluids drained from endobronchial tubes during video-assisted thoracic surgery (VATS) for

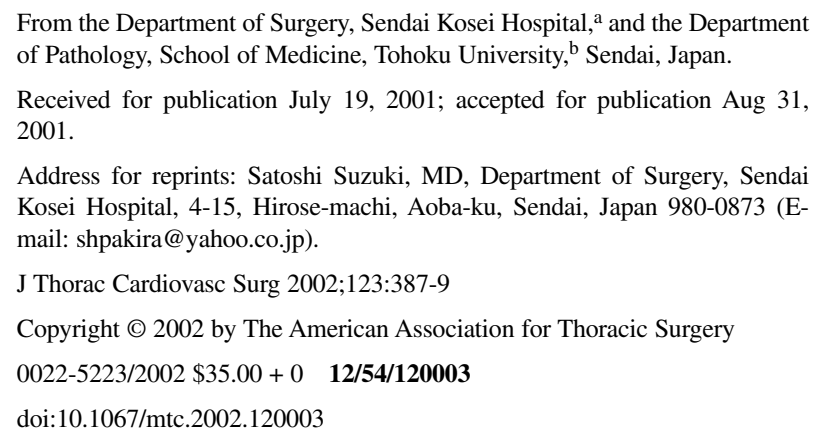

From the Department of Surgery, Sendai Kosei Hospital, ${ }^{a}$ and the Department

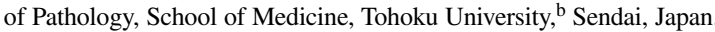

Received for publication July 19, 2001; accepted for publication Aug 31, 2001.

Address for reprints: Satoshi Suzuki, MD, Department of Surgery, Sendai Kosei Hospital, 4-15, Hirose-machi, Aoba-ku, Sendai, Japan 980-0873 (Email: shpakira@yahoo.co.jp).

J Thorac Cardiovasc Surg 2002;123:387-9

Copyright $\odot 2002$ by The American Association for Thoracic Surgery

$0022-5223 / 2002 \$ 35.00+0 \quad \mathbf{1 2 / 5 4 / 1 2 0 0 0 3}$

doi: $10.1067 / \mathrm{mtc} .2002 .120003$

pneumothoraces. We examined edema fluids and histologic characteristics of the lung and provide evidence of acute inflammatory response and active $\mathrm{Na}^{+}$transport in the process of rapid development and resolution of reexpansion PE.

\section{Clinical Summary}

PATIENT 1. A 52-year-old man had shortness of breath for 10 days. The chest $\mathrm{x}$-ray film showed a collapsed left lung with a giant bulla. The left upper lobe remained completely collapsed after overnight chest drainage. A double-lumen endobronchial tube was inserted, and both lungs were ventilated at a maximal airway pressure of $20 \mathrm{~cm} \mathrm{H}_{2} \mathrm{O}$. Approximately 1 hour after the start of mechanical ventilation, yellowish and clear fluid drained from the left lung over 30 minutes ( $20 \mathrm{~mL}$ in total). Pulse oximetry was consistently at $100 \%$ with the patient breathing $50 \%$ oxygen. Once the drainage of fluid subsided, the giant bulla was resected by VATS. A lung tissue sample was also resected from the edematous lingual segment. A postoperative chest x-ray film showed parenchymal infiltration only in the reexpanded lung lobe (Figure 1). A chest $x$-ray film on the third postoperative day showed resolution of pulmonary edema. 


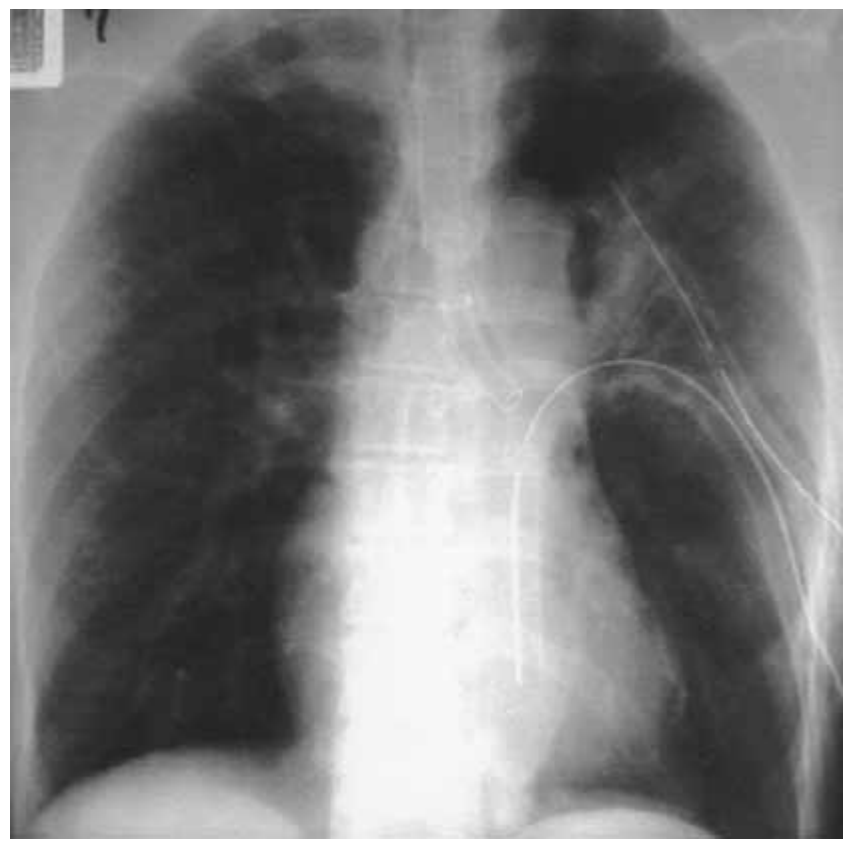

Figure 1. Postoperative chest x-ray films of case 1. Very fine parenchymal infiltration was seen only in the reexpanded lung lobe.

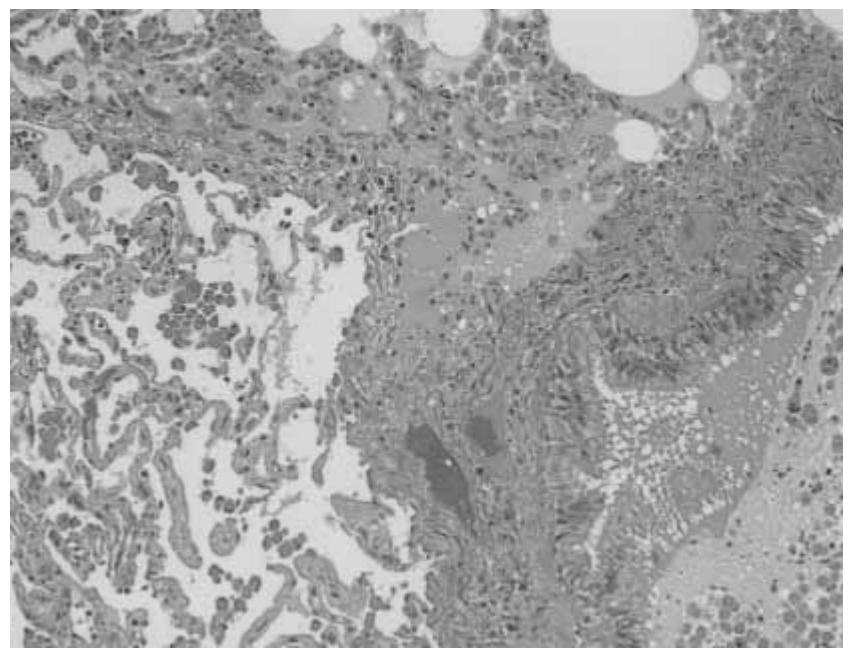

Figure 2. Histologic features of the lung in case 2. The alveolar spaces were mostly fluid-free and only fine protein debris was found. Small airways were filled with protein-rich fluids. (Hematoxylin and eosin; original magnification $\times \mathbf{2 0 0}$.)

PATIENT 2. A 30-year-old man had sustained pain in the right side of the chest and shortness of breath for more than a week. A chest $\mathrm{x}$-ray film showed a collapsed right lung with a small bulla in the upper lobe. Overnight chest drainage revealed continuous air leakage, and the right upper lobe remained collapsed. A doublelumen endobronchial tube was inserted, and both lungs were ventilated at a maximal airway pressure of $20 \mathrm{~cm} \mathrm{H}_{2} \mathrm{O}$. Approximately
TABLE 1. Laboratory data of tracheal aspirates

\begin{tabular}{|c|c|c|}
\hline & $\begin{array}{c}\text { Patient } 1 \\
\text { (fluid/serum) }\end{array}$ & $\begin{array}{c}\text { Patient } 2 \\
\text { (fluid/serum) }\end{array}$ \\
\hline Total protein (g/dL) & $6.4 / 6.1$ & $8.1 / 6.4$ \\
\hline Albumin (g/dL) & $4.4 / 3.8$ & $5.3 / 3.6$ \\
\hline $\mathrm{Na}^{+}(\mathrm{mmol} / \mathrm{L})$ & $142 / 142$ & $138 / 143$ \\
\hline $\mathrm{K}^{+}(\mathrm{mmol} / \mathrm{L})$ & $5.5 / 4.3$ & 7.8/3.8 \\
\hline $\mathrm{Cl}^{-}(\mathrm{mmol} / \mathrm{L})$ & $110 / 105$ & $102 / 109$ \\
\hline Lactate dehydrogenase (U/L) & $730 / 280$ & $2773 / 409$ \\
\hline Interleukin 6 (pg/mL) & $3100 / 3.9$ & $12,300 / 15.6$ \\
\hline Interleukin 8 (pg/mL) & $773 /<12.5$ & $1260 /<12.5$ \\
\hline GM-CSF* (pg/mL) & Not measured & $30 / 8$ \\
\hline Granulocyte elastase $(\mu \mathrm{g} / \mathrm{mL})$ & Not measured & $2760 / 107$ \\
\hline
\end{tabular}

*Granulocyte macrophages-colony stimulating factor.

1 hour after starting mechanical ventilation, yellowish and clear fluid (10 $\mathrm{mL}$ in total) drained from the right lung for 30 minutes. Pulse oximetry was consistently at $100 \%$ with the patient breathing 50\% oxygen. Once fluid drainage subsided, a bulla at the edematous right upper lobe was resected by VATS. A postoperative chest $\mathrm{x}$-ray film showed parenchymal infiltration only in the reexpanded lung lobe. A chest x-ray film revealed clearing of the edema on the second postoperative day.

Total protein and albumin concentrations in edema fluids were higher than those in serum, whereas $\mathrm{Na}^{+}$and $\mathrm{Cl}^{-}$concentrations were similar. Levels of inflammatory cytokines such as interleukin 8 were also markedly elevated in the face of the almost normal levels in serum (Table 1). The cell count in edema fluid of patient 1 was 250 cells $/ \mathrm{mm}^{3}$, with $49 \%$ neutrophils, $9 \%$ lymphocytes, and $42 \%$ macrophages.

Light microscopic examination of the resected lung tissues showed markedly dilated lymphatic vessels in the pleura and perivascular area. However, the alveolar spaces were mostly fluidfree and only fine protein debris was found. Protein-rich fluids existed in small airways (Figure 2).

\section{Discussion}

High protein concentration in edema fluids indicates that our 2 cases of reexpansion PE were examples of high-permeability edema. In addition, neutrophils and high levels of inflammatory cytokines in the fluids suggest that an acute inflammatory response took place in the reexpanded lung. ${ }^{2}$ Inflammatory cytokines such as interleukin 8 activate neutrophils and alter permeability of the alveolar-capillary barrier. However, we found total protein and albumin at higher concentrations than those in serum, suggesting that lung edema fluids had been condensed before collection. This is different from our previous observation in reexpansion PE during thoracotomy, which was performed to remove gigantic intrathoracic sarcomas. ${ }^{2}$ We found high protein concentration in lung edema fluids; however, the fluid/blood concentration ratio did not exceed $1 .^{2}$ Inasmuch as there were no differences in $\mathrm{Na}^{+}$or $\mathrm{Cl}^{-}$concentrations between edema fluids and serum, it is very unlikely that the fluids simply evaporated in the endobronchial tube. Rather, lung edema fluids might be absorbed in part from the alveolar spaces. Histologic studies of the lung in both cases showed interstitial 
edema, whereas the alveolar spaces were mostly fluid-free when the small airways were filled with protein-rich edema fluids.

Fluid absorption from the alveolar spaces may depend in part on active $\mathrm{Na}^{+}$transport of the alveolar epithelium. ${ }^{3}$ Resected human lungs are known to display active $\mathrm{Na}^{+}$transport with alveolar fluid clearance at a rate of $12 \%$ in 4 hours. ${ }^{4}$ Active $\mathrm{Na}^{+}$transport produces fluid movement from the alveolar spaces into the interstitium and lymphatic vessels. Sieving of larger molecules at the alveolar epithelium may then increase albumin concentration in the air space without significant changes in electrolyte concentrations. Assuming that albumin concentrations in the air space were equal to serum at the onset of reexpansion PE, lung edema fluids were roughly calculated to be concentrated to $1: 1.16(86 \%)$ and $1: 1.47(68 \%)$ in volume over 90 minutes, giving $9 \%$ and $21 \%$ per hour clearance of alveolar fluid in patients 1 and 2, respectively. The faster clearance rate in our cases of reexpansion PE may be explained by the presence of blood flow and mechanical ventilation. The latter may increase the alveolar surface area and acceler- ate fluid movement across the alveolar epithelium. Continuous positive airway pressure is reported to be beneficial in the treatment of reexpansion $\mathrm{PE} .^{5}$

\section{References}

1. Mahfood S, Hix WR, Aaron BL, Blaes P, Watson DC. Reexpansion pulmonary edema. Ann Thorac Surg. 1997;63:1206-7.

2. Suzuki S, Tanita T, Koike K, Fujimura S. Evidence of acute inflammatory response in reexpansion pulmonary edema. Chest. 1992;101: 275-6.

3. Matthay MA, Wiener-Kronish JP. Intact epithelial barrier function is critical for the resolution of alveolar edema in humans. Am Rev Respir Dis. 1990;142:1250-7.

4. Sakuma T, Okaniwa G, Nakada T, Nishimura T, Fujimura S, Matthay MA. Alveolar fluid clearance in the resected human lungs. Am $J$ Respir Crit Care Med. 1994;150:305-10.

5. Iqbal M, Multz AS, Rossoff LJ, Lackner RP. Reexpansion pulmonary edema after VATS successfully treated with continuous positive airway pressure. Ann Thorac Surg. 2000;70:669-71. 